\title{
Hepatic Portal Venous Gas
}

\author{
Yasushi Ito and Yasushi Komatsu
}

Key words: portal gas, vomiting, hypokalemia

(Intern Med 51: 2665, 2012)

(DOI: 10.2169/internalmedicine.51.8428)
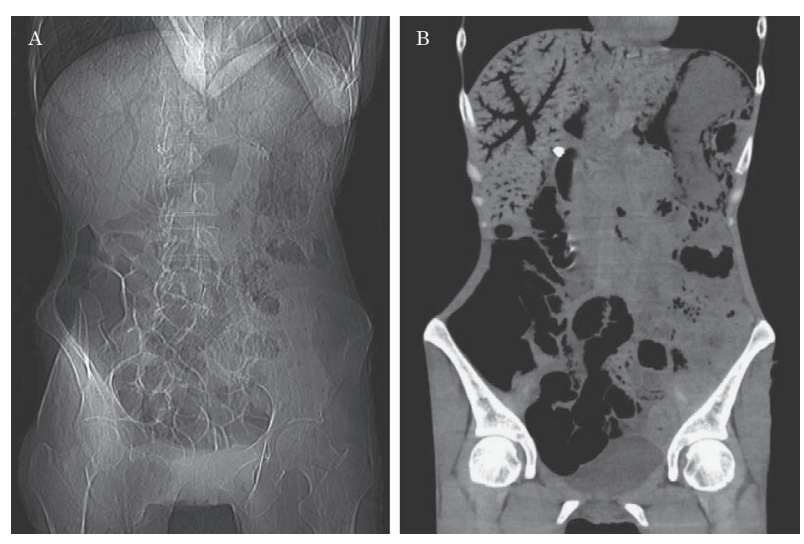

Picture

A 42-year-old woman with bulimia nervosa presented to the emergency room with increasing weakness and vomiting. The physical examination was remarkable for her weight of $26.1 \mathrm{~kg}$, distended but non-tender abdomen with decreased bowel sounds. Laboratory tests revealed serum sodium of $124 \mathrm{mmol} / \mathrm{L}$, potassium of $1.6 \mathrm{mmol} / \mathrm{L}$, magnesium of $1.4 \mathrm{mmol} / \mathrm{L}$ and a leucocyte count of $5.5 \times 10^{9} / \mathrm{L}$. A computed tomography (CT) scan of the abdomen showed marked gas in the portal system, gastric wall and intestinal lumen (Picture A and B). The presence of hepatic portal gas in abdominal radiographs was once thought to be an ominous sign and considered an indication for an emergency laparotomy. The development of CT has created more opportunities to visualize such cases and conservative management is increasingly recognized as being appropriate in many instances especially when signs of bowel ischemia are absent (1). The present patient was transferred to a tertiary care center with a psychiatry unit, treated reportedly conservatively and subsequently discharged 10 days later.

The authors state that they have no Conflict of Interest (COI).

\section{Reference}

1. Nelson AL, Millington TM, Sahani D, et al. Hepatic portal venous gas: the ABCs of management. Arch Surg 144: 575-581, 2009. 